\title{
Research on Financial Management System in Cloud Computing Zhengang $\mathrm{Yao}^{1,}$, , Jinxin Yuan ${ }^{1, a}$ \\ ${ }^{1}$ Suzhou Polytechnic Institute of Agriculture, Suzhou 215000, China \\ ayzgkeyan@163.com
}

Keywords: Management system, cloud computing, financial management

\begin{abstract}
A cloud computing based financial management system was proposed in this paper. According to the needs, system was constructed by client, management server and cloud server. As the core functions, insert function, delete function, query function and synchronize function were designed.
\end{abstract}

\section{Instroduction}

Low personal savings rates of college students have amplified the calls for financial management [1]. Personal cloud is a collection of digital content and services which are accessible from any device [2]. A cloud computing based financial management system can be used to promote financial management skills of the college students. A survey of the needs of the financial management system was done to 300 students and found that $89 \%$ of them chose to use the system on mobile equipment. The system should record the income and expenditure for the students. Budget Management is based on the statistical reports on the income and expenditure.

\section{System Architecture}

According to the needs, system is constructed by client, server and cloud server. Client is based on android platform and a cross-platform MVC framework is used. Client integrates XML module, HTTP transmission module and Service Pack module. Data is translated in xml format using the SSL protocol [3]. The protocol allows client/server applications to communicate in a way that cannot be eavesdropped. The server is constructed by link module, application module and management module. Link module is based on Mule ESB platform. Services are created and managed in this module. The reused services are managed by Mule ESB container. Service decomposition, information routing and data translation are the major functions of this module. Business logic is separated from the service. Then the interface was called locally. The protocol and format are hidden for security. Data can be combined, sorted and filtered by the rules and context. Data should be transformed between different formats by using different protocols. Application module is based on EJB3. Persistence, transactional integrity and security are handled in a standard way. Management module is based on Liferay. Application portal is based on android platform and Struts framework is used for the user interface. Web service is used for remote invoke and Lucene is used for full-text retrieval. All the services are established in the cloud server. For different requirements, different web services are called remotely. The architecture is shown in Fig1.
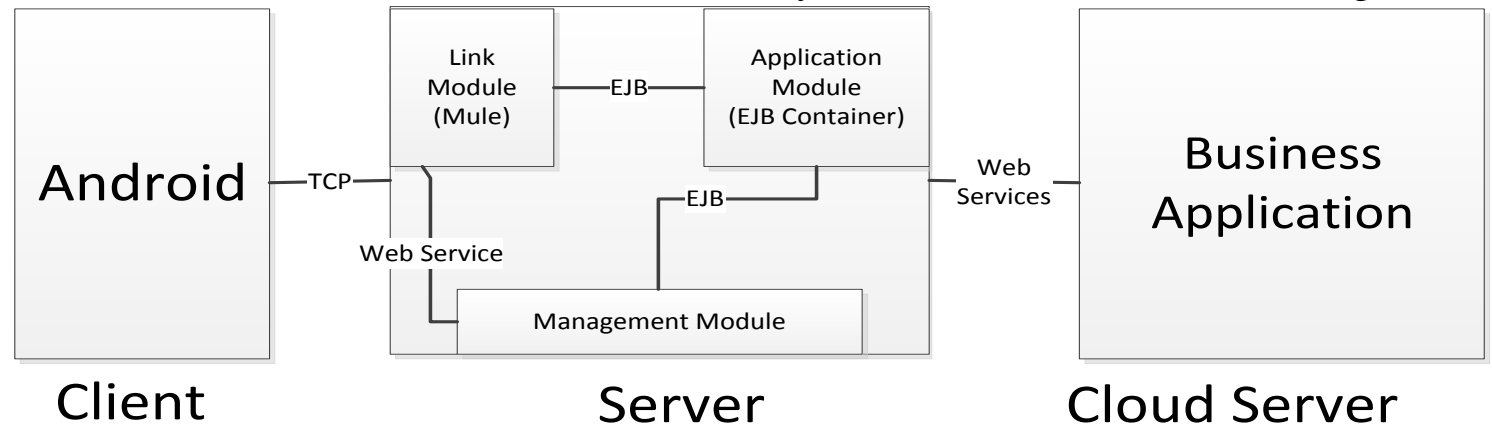

Fig.1 System architecture 


\section{Core Functions}

Insert Function. It is shown in Fig2.After login the system, user may select insertion to obtain detailed information by user input, like the date, event, amount, type and classification. The insert method will be called to insert detail data into the table in the database. If the input is not valid or incomplete, a prompt is made to ask the user to re-enter.

Delete Function. It is shown in Fig3.After choosing the details of the list, including monthly and daily list of income and expenditure details, users can select the item in the list to be deleted. After the selection, the delete method is called to delete the data from the database.

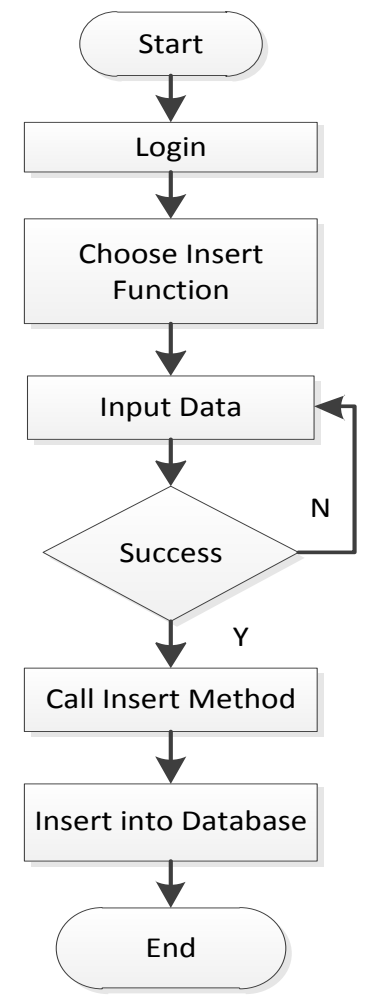

Fig.2 Insert function

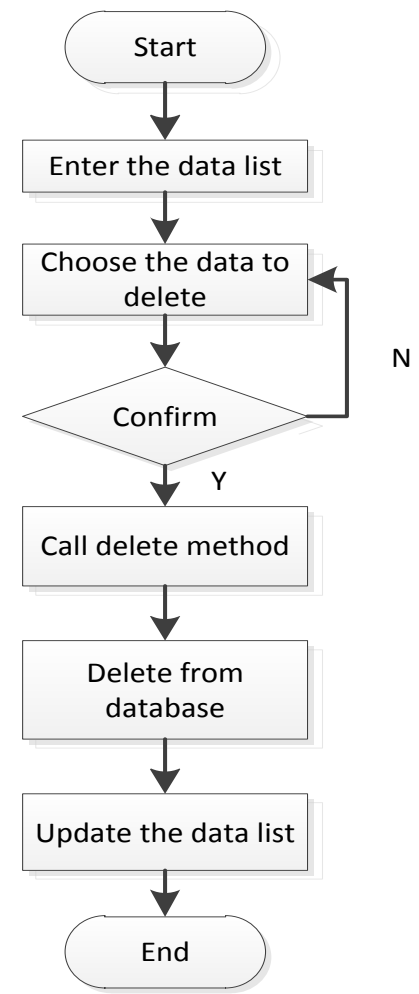

Fig.3 Delete function

Query Function. It is show in Fig4.Query function contains query data by month, day and types. The main query condition is date which is input by users. When query by month, the system calls query method to obtain details of the month. The monthly summary information is displayed through the interactive interface controls, and specific details of each month are displayed when the user chooses. When query by day, the date should be entered, and the query method will return a list of daily details. Classification query to account for statistical month period, after user inputs years, query method is called to obtain the data list. While interacting with the user through the confirmation, the monthly balance of payments data will be displayed in the form of a pie chart or histogram.

Synchronize Function. It is shown in Fig5.Synchronize function is obtained by mobile equipment and computers. Mobile terminals and computers build a connection mode. There is a database which has the same tables in the computer. Mobile terminal maintain a synchronized signal in a file. After the user activates the synchronization function, the phone and the computer will establish an available link. The second step is to send the synchronization number to the terminal server. There is a table that records the synchronization numbers. If the synchronization number is not larger than the number that is in the database of computer the synchronization has been completed, and the information will be returned to the mobile terminal and the link will be closed. If the synchronization number is greater than the exist number, the records will be calculated. Then the server will transmit those records to the mobile terminals. After the mobile terminals received these records, the database will be updated. 


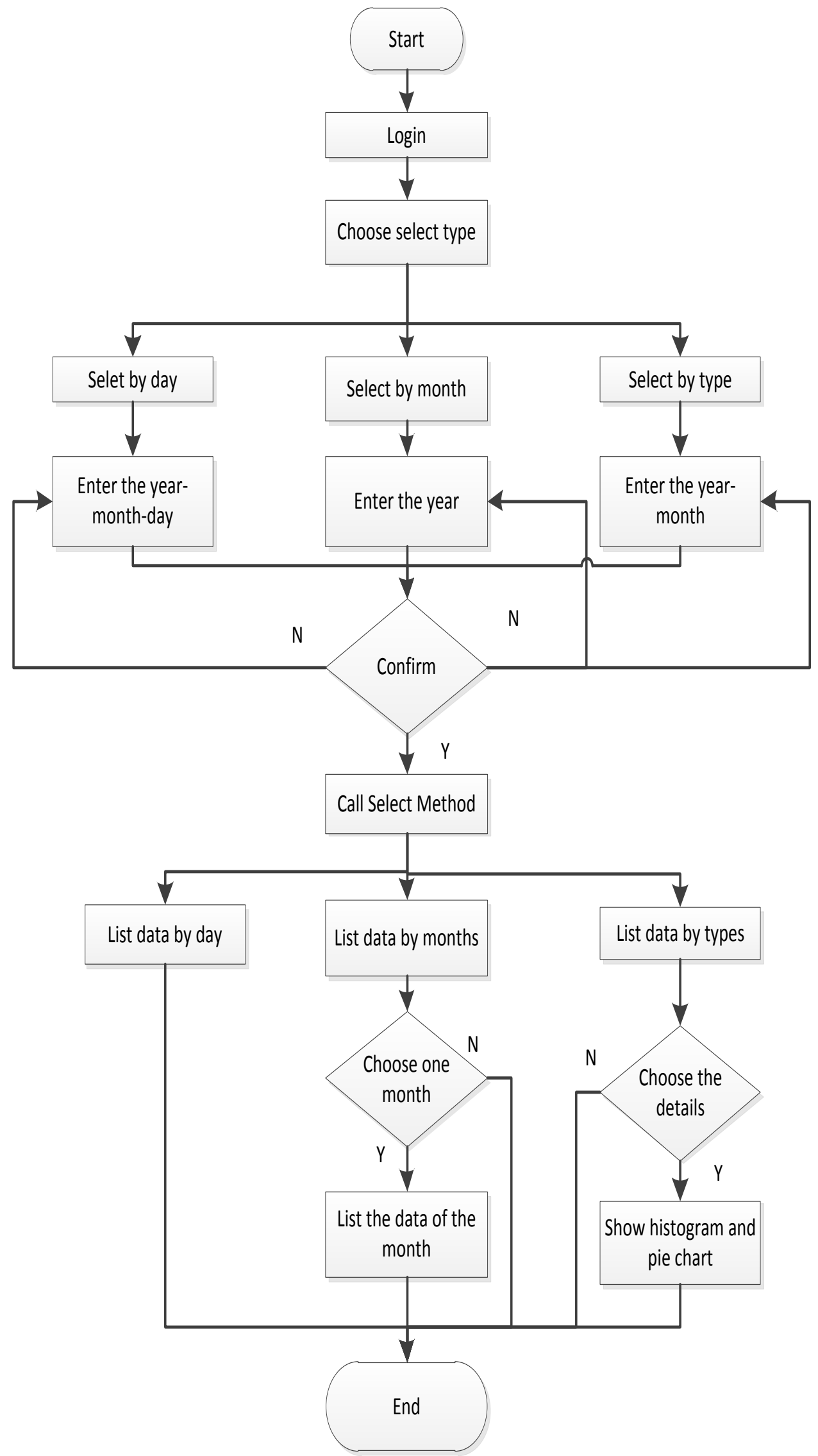

Fig.4 Query function 


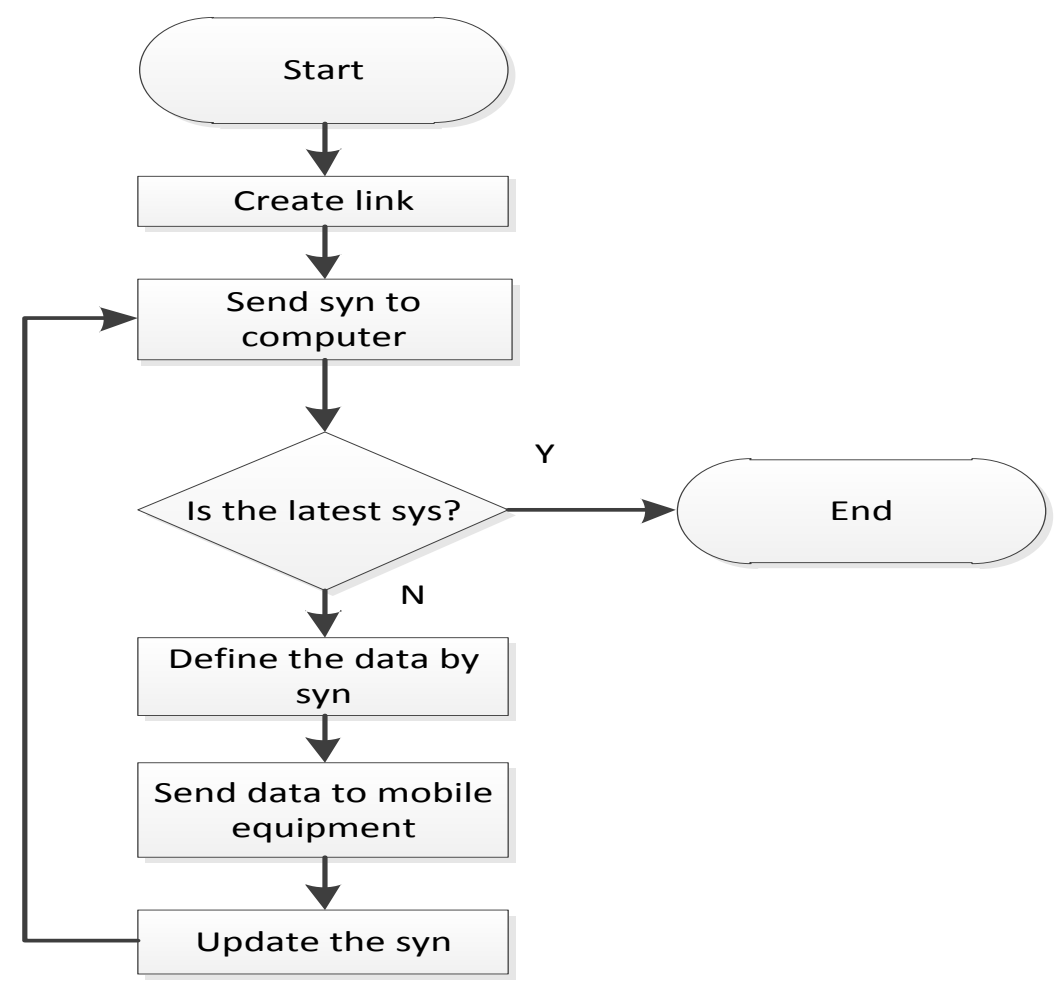

Fig.5 Synchronize function

\section{Results Analysis}

After constructing the financial management system, the test results were shown in Table 1. Test results of synchronize function between mobile equipment and computers meet the actual demand. Table 1 Analysis of synchronize function

\begin{tabular}{cccc}
\hline No. & Send Data[K] & Received Data $[\mathrm{K}]$ & Percentage(S/R) \\
\hline 1 & 10 & 10 & $100 \%$ \\
\hline 2 & 100 & 100 & $100 \%$ \\
\hline 3 & 1000 & 1000 & $100 \%$ \\
\hline
\end{tabular}

\section{Conclusion}

Insert function, delete function, query function and synchronize function are the core functions of the financial management system. The system architecture and the functions which are designed in this paper are efficient and practical. This cloud computing based financial management system can be used to promote financial management skills of the college students.

\section{Acknowledgements}

Jiangsu Students Innovation and Entrepreneurship Training Program: 201412808015Y.

\section{References}

[1] Kim H, De Vaney S A: The Effect of Personal Financial Management, Bank Relationship and Small Business Characteristics on Small Business Productivity[C].Vol.57 (2011), p.184.

[2] Hari A, Viswanathan R, Lakshman T V: The Personal Cloud-Design, Architecture and Matchmaking Algorithms for Resource Management[C].Vol.1 (2012), p.384

[3] Hickman K, Elgamal T: The SSL protocol [J].Vol. 1(1995), p.501. 